\title{
Volume-based solvation models out-perform area-based models in combined studies of wild-type and mutated protein-protein interfaces

\author{
Salim Bougouffa and Jim Warwicker*
}

Address: Faculty of Life Sciences, the Michael Smith Building, University of Manchester, Oxford Road, Manchester, M13 9PT, UK

Email: Salim Bougouffa - salim.bougouffa@postgrad.manchester.ac.uk; Jim Warwicker* - jim.warwicker@manchester.ac.uk

* Corresponding author

Published: 21 October 2008

BMC Bioinformatics 2008, 9:448 doi:10.1 186/1471-2105-9-448

This article is available from: http://www.biomedcentral.com/I47/-2/05/9/448

(c) 2008 Bougouffa and Warwicker; licensee BioMed Central Ltd.

This is an Open Access article distributed under the terms of the Creative Commons Attribution License (http://creativecommons.org/licenses/by/2.0), which permits unrestricted use, distribution, and reproduction in any medium, provided the original work is properly cited.

\begin{abstract}
Background: Empirical binding models have previously been investigated for the energetics of protein complexation ( $\Delta \mathrm{G}$ models) and for the influence of mutations on complexation (i.e. differences between wild-type and mutant complexes, $\Delta \Delta \mathrm{G}$ models). We construct binding models to directly compare these processes, which have generally been studied separately.

Results: Although reasonable fit models were found for both $\Delta \mathrm{G}$ and $\Delta \Delta \mathrm{G}$ cases, they differ substantially. In a dataset curated for the absence of mainchain rearrangement upon binding, nonpolar area burial is a major determinant of $\Delta \mathrm{G}$ models. However this $\Delta \mathrm{G}$ model does not fit well to the data for binding differences upon mutation. Burial of non-polar area is weighted down in fitting of $\Delta \Delta \mathrm{G}$ models. These calculations were made with no repacking of sidechains upon complexation, and only minimal packing upon mutation. We investigated the consequences of more extensive packing changes with a modified mean-field packing scheme. Rather than emphasising solvent exposure with relatively extended sidechains, rotamers are selected that exhibit maximal packing with protein. This provides solvent accessible areas for proteins that are much closer to those of experimental structures than the more extended sidechain regime. The new packing scheme increases changes in non-polar burial for mutants compared to wild-type proteins, but does not substantially improve agreement between $\Delta \mathrm{G}$ and $\Delta \Delta \mathrm{G}$ binding models.
\end{abstract}

Conclusion: We conclude that solvent accessible area, based on modelled mutant structures, is a poor correlate for $\Delta \Delta G$ upon mutation. A simple volume-based, rather than solvent accessibilitybased, model is constructed for $\Delta \mathrm{G}$ and $\Delta \Delta \mathrm{G}$ systems. This shows a more consistent behaviour. We discuss the efficacy of volume, as opposed to area, approaches to describe the energetic consequences of mutations at interfaces. This knowledge can be used to develop simple computational screens for binding in comparative modelled interfaces.

\section{Background}

Macromolecular complexation is key to many biological processes, and has been a subject of experimental study for many decades. The last few years have seen significant advances in high throughput detection of protein-protein interactions, for example with yeast two hybrid [1] and affinity purification methods feeding into analysis by mass spectrometry [2]. These laboratory advances have 
led to a new area of bioinformatics analysis, interpreting the data in terms of interaction networks, and putting such networks in a biological context [3]. At the same time, structural biology continues to visualise interfaces at atomic resolution $[4,5]$, whilst computational biology addresses whether proteins can be docked into the correct complexes $[6,7]$, and develops models for the prediction of binding affinities [8]. These are fundamental questions, combining the physico-chemical properties of atomic interactions with biological activity.

Docking of two proteins is determined by complementarity of shape and of pairwise interactions within shapematched patches [9]. Methods for predicting mainchain alteration are still relatively poor, so that successful docking methods have been largely restricted to proteins for which there is little conformational change between the complexed and uncomplexed forms [10]. The issue of sidechain rotameric variation upon complexation can also cause problems [11], necessitating the development of methods to sample sidechain conformers [12]. There are promising advances in the handling of conformational variation, for both sidechains and mainchain, which simulate variation and show that the correct solution can be identified in a cluster of well-packed configurations $[13,14]$.

Computational research in protein docking inevitably overlaps studies that construct models for binding affinities, through the common use of force-fields. One particularly important growth area is the assessment of binding potential for proteins that are homologous with the constituents of characterised complexes i.e. comparative modelling of complexes based on known interfaces $[15,16]$. Whereas comparative modelling of individual domains based on homology demonstrates the fold for the sequence of interest, albeit with variations that may not be easy to model, similar modelling applied separately to non-covalently linked components must address the question of whether a viable interface is maintained. If effective algorithms can be developed in this area, then structural bioinformatics, combined with structural biology, will be a valuable complement to the high throughput experimental methods for determining proteinprotein interactions. The question of what features determine interfacial stability had been extensively studied. A recurring theme is the importance, in many cases, of buried non-polar area [17]. This simple observation, along with the complexity and computational scale of attempts to calculate free energies of binding by simulation, has led to the development of empirical binding models [8]. These involve the separation into terms that each represent some physical feature or combination of features, and which are linearly combined and the relevant weights determined through fitting to a test set of experimental data. Terms reflect properties such as: the hydrophobic effect/buried non-polar surface area; electrostatic interactions; van der Waals interactions; sidechain rotameric entropy; rotational and translation entropy of complexation. The limitations of such models, especially the lack of a simulation component, are counteracted by their potential to detect trends through fitting to experimental data.

There is also widespread interest in modelling the influence of mutations at interfaces, which in biological terms could map for example to the role of single nucleotide polymorphisms in complexes [18]. Binding models have been derived from fitting to measured differences in binding energies for mutant complexes relative to wild-type $[19,20]$. In the current work, we denote models for binding in complexes as $\Delta \mathrm{G}$ models, and those for differences (upon mutation) between complexes as $\Delta \Delta \mathrm{G}$ models. Since $\Delta G$ and $\Delta \Delta G$ models use the same range of terms, apart from cancellation of rotational/translational entropy, it is of interest to compare them. This is the subject of the current study.

An important property of interfaces is the degree to which conformation changes between the complexed and uncomplexed forms. We have restricted our dataset of wild-type proteins to those for which there is no evidence in the literature for mainchain changes, and for mutant proteins the assumption is made that the backbone does not change. With regard to sidechains, we make use of a mean field technique adapted previously in our laboratory [21], from earlier work [22]. With this method the probability of a rotamer is higher where it can fit with a larger number of neighbouring sidechain rotamers, at a given van der Waals tolerance. Thus higher probability values, in this framework, tend to select rotamers with higher solvent accessibility, and conversely lower probability generally maps to lower solvent accessibility. Rotamer prediction is more difficult for sidechains without structural constraints [23], and prediction accuracy reduces in these cases for commonly-used methods such as SCWRL [24]. In the current work we study the effect of differential modelling of interfacial changes, using either the higher solvent accessibility scheme ("HighSA"), or in the lower solvent accessibility ("LowSA") scheme. Comparison with accessible solvent areas in experimental structures suggests that the more compact, LowSA, configurations may give a more appropriate representation.

In addition to the question of how to repack sidechains, there is also the issue of which sidechains to repack. In a minimal scheme for repacking, using the experimental rotamers where possible, wild-type complexes are not repacked at all, and mutations are modelled simply by rotamer selection for the mutated amino acid. Such structural models we denote as "Minimal". In contrast, it is 
also possible to repack all sidechains for all molecules, so that the packing can (in principle) change upon complexation and/or upon mutation. We label such schemes as "Complete". Thus Minimal-HighSA packed wild-type complexes in $\Delta \mathrm{G}$ models are simply the structures from the experimentally-determined complexes, and for mutations in $\Delta \Delta \mathrm{G}$ models would simply be modelling of the mutated residue with the most solvent accessible rotamer, in the free and complexed states. Complete-LowSA takes low solvent accessibility rotamers, and repacks all sidechains, in both free and uncomplexed states.

Our results show that the importance of buried non-polar surface for $\Delta G$ models is not reflected in $\Delta \Delta G$ models, when using HighSA packing (Minimal or Complete). We examine the effect of Complete-LowSA packing, allowing for greater environment-specific relaxation. Although the magnitude of calculated non-polar burial differences between wild-type and mutants is increased, the Complete-LowSA packing does not significantly improve consistency between $\Delta \mathrm{G}$ and $\Delta \Delta \mathrm{G}$ models. A key factor in the inconsistency is that changes in non-polar burial upon mutation can be either positive or negative, in comparison with the experimental $\Delta \Delta G$ values in our dataset that are almost exclusively unfavourable upon mutation. This is true for all packing schemes used, presumably reflecting inaccuracies in predicting conformation upon mutation and/or that structure is more fluid than can be represented by single conformers.
The inconsistency of $\Delta \mathrm{G}$ and $\Delta \Delta \mathrm{G}$ models is substantially reduced when a simple volume-based representation of interfacial changes replaces the area-based description. We discuss this result in the context of comparative modelling for protein-protein interfaces.

\section{Results and discussion \\ Non-polar surface area dominates $\Delta G$ binding models (no sidechain repacking)}

A set of protein-protein complexes with known structures and binding energies was collated as described in the Methods section. We ascertained that there was no evidence for substantial mainchain conformational change in these complexes through a survey of the literature. Although structures for the uncomplexed components were not used in calculations, in several cases these were used for assessment of conformational change. Table 1 lists the set of wild-type complexes used in the current study, together with their complexation energies.

Figure 1 shows best fit models for the wild-type complexes, setting $\Delta \mathrm{G}_{\text {ROT-TRANS }}$ to zero. Table 2 gives the model weights corresponding to Figure 1, and with models calculated for $\Delta \mathrm{G}_{\text {ROT-TRANS }}=10 \mathrm{~kJ} / \mathrm{mole}$, investigating the range derived from experiment [25]. There is very little difference in models at 0 or $10 \mathrm{~kJ} / \mathrm{mole}$ for $\Delta \mathrm{G}_{\text {ROT-TRANS' }}$ and this is generally true throughout this work. The Methods section describes modelling of $\Delta \mathrm{G}_{\mathrm{SC} \text {-ROT }}$ according to a Locked scheme (sidechains free in separated components and fixed in the interface), and an Unlocked scheme (free

Table I: Wild-type complexes and binding energies.

\begin{tabular}{|c|c|c|c|c|}
\hline Protein A & Protein B & $\Delta \mathbf{G}(\mathbf{k} / /$ mole $)$ & PDB & Ref \\
\hline BPTI & Chymotrypsin & -44.96 & ICBW & [49] \\
\hline Barnase & Barstar & -79.50 & IB27 & {$[50]$} \\
\hline Subtilisin Carlsberg & OMTKY3 & -59.31 & IROR & {$[5 \mathrm{I}]$} \\
\hline RaplA & Rafl & -35.98 & ICIY & {$[52]$} \\
\hline Ras & Byr2 & -38.45 & IK8R & {$[53]$} \\
\hline Fv DI.3 & Fv E5.2 & -45.48 & IDVF & {$[54]$} \\
\hline Fv DI.3 & HEWL & -45.10 & IVFB & {$[55]$} \\
\hline BPTI & Trypsin & -75.16 & 2PTC & [49] \\
\hline HyHELIO Fab & HEWL & -56.21 & $3 \mathrm{HFM}$ & [56] \\
\hline RalGDS & Ras & -35.15 & ILFD & [57] \\
\hline Subtilisin Carlsberg & Eglin C & -54.76 & ICSE & [58] \\
\hline IM9 & Colicin E9 & -78.62 & IEMV & [59] \\
\hline HyHEL5 Fab & HEWL & -59.36 & IYQV & {$[60]$} \\
\hline SGPB & OMTKY3 & -61.45 & $3 S G B$ & {$[61]$} \\
\hline Ribonuclease Inhibitor & Angiogenin & -87.15 & IA4Y & {$[62]$} \\
\hline N9 Neuraminidase & NCIO Fab & -48.50 & INMB & [63] \\
\hline Subtilisin BPN' & SSI & -61.33 & $2 \mathrm{SIC}$ & [64] \\
\hline Thrombin & Thrombomodulin & -53.09 & IDX5 & {$[65]$} \\
\hline Ribonuclease A & Ribonuclease Inhibitor & -76.30 & IDFJ & {$[62]$} \\
\hline Kallikrein A & BPTI & -51.83 & $2 \mathrm{KAI}$ & [66] \\
\hline
\end{tabular}

Protein constituents of the complexes are given, with the following abbreviations: OMTKY3, turkey ovomucoid third domain; HEWL, Hen Egg White Lysozyme; BPTI, Bovine Pancreatic Trypsin Inhibitor; IM9, Immunity Protein 9; SGPB Streptomyces griseus protease B; SSI, Streptomyces Subtilisin Inhibitor. 
(a)

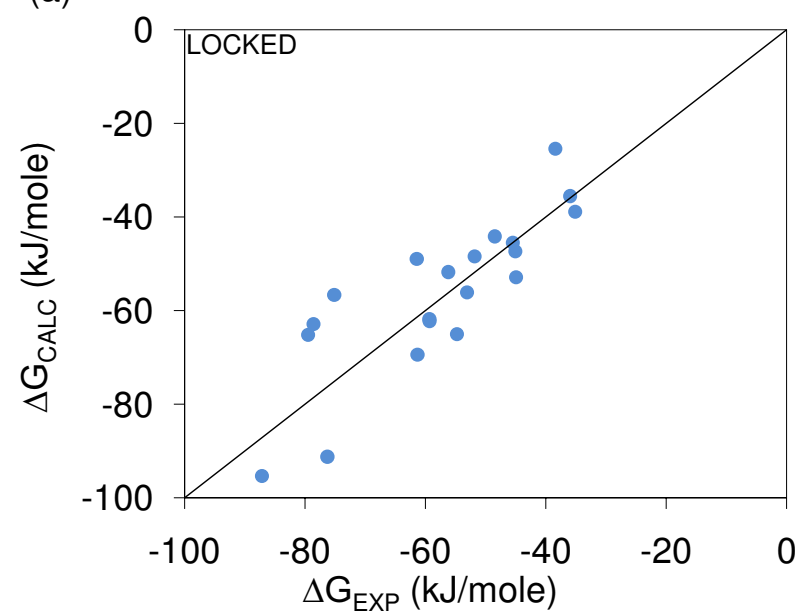

(c)

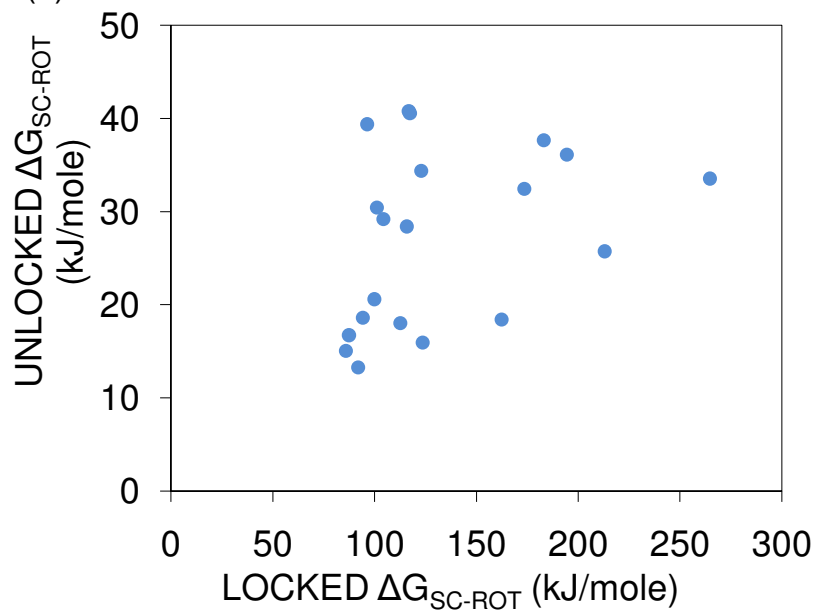

(b)

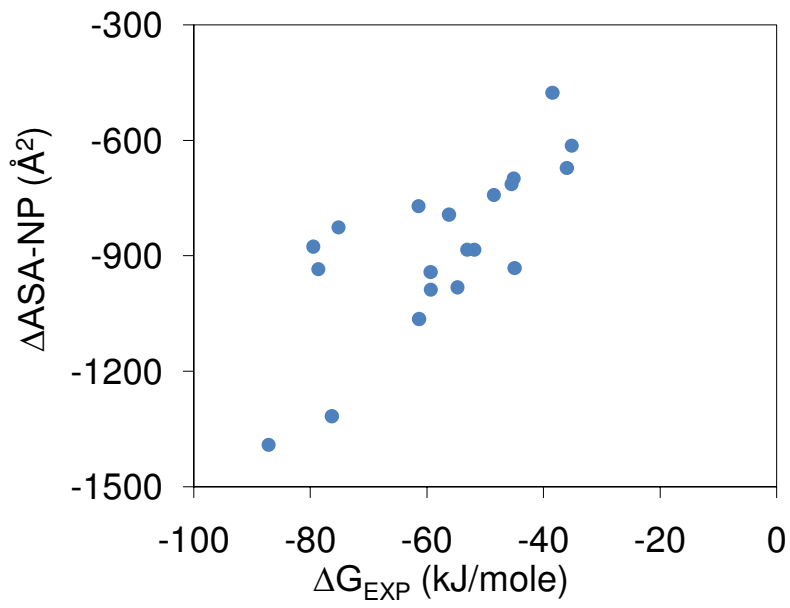

(d)

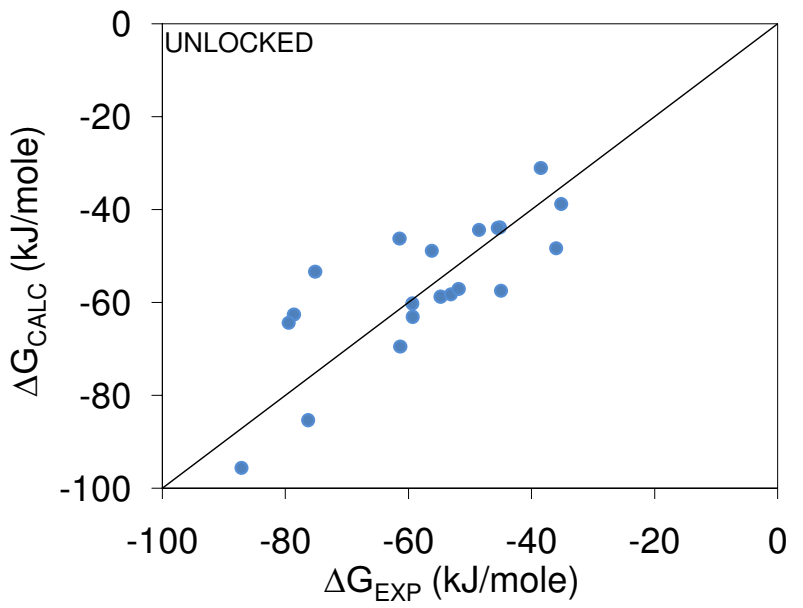

Figure I

$\Delta \mathbf{G}$ models with Locked and Unlocked interfacial sidechains, Minimal-HighSA. Binding models are fit to wild-type $\Delta G_{\text {EXP }}$, and Minimal-HighSA implies no repacking for wild-type systems. (a) Model fit with $\Delta G_{S C-R O T}$ calculated for Locked interfacial sidechains and $\Delta G_{R O T-T R A N S}=0\left(R^{2}=0.69\right)$. (b) Correlation between $\Delta G_{\text {EXP }}$ and $\Delta(A S A-N P)\left(R^{2}=0.55\right)$. (c) Poor correlation $\left(R^{2}=0.12\right)$ between $\Delta G_{S C-R O T}$ calculated with Locked and Unlocked interfacial sidechains. (d) Model fit with $\Delta \mathrm{G}_{\mathrm{SC} \text { - }}$ ROT Calculated for Locked interfacial sidechains and $\Delta G_{R O T \text {-TRANS }}=0\left(R^{2}=0.6 \mathrm{I}\right)$. For plots with equivalent $y$ and $x$ quantities, the line $y=x$ is shown.

at all points). Comparing Figure 1 panels (a) and (d), and also Table 2, whilst there are some differences between the Locked and Unlocked interfacial sidechain models for $\Delta \mathrm{G}_{\mathrm{SC}-\mathrm{ROT}}$, with somewhat better performance for the Locked case, the overall domination by $\Delta \mathrm{G}_{\mathrm{ASA}-\mathrm{NP}}$ is common. Figure $1(\mathrm{c})$ shows that Locked $\Delta \mathrm{G}_{\mathrm{SC}-\text { ROT }}$ values are, as expected, substantially larger than the Unlocked sidechain $\Delta \mathrm{G}_{\mathrm{SC}-\mathrm{ROT}}$ values, but with little correlation between them. Table 2 indicates that the best fit model uses a negative (unphysical) weight for Unlocked interfacial sidechain $\Delta \mathrm{G}_{\mathrm{SC}-\mathrm{ROT}}$. Generally, several of the weights reported in Table 2 are negative. Where these are of small magnitude they will have little contribution to a binding model, and larger magnitude negative weights are likely to result from anti-correlation with other features that have large and positive weights. Table 3 shows correlations between calculated properties, and between calculated properties and experimental binding energies. The correlation shown in Figure 1(b) and the weights in Table 2 make it clear that buried non-polar area dominates the $\Delta \mathrm{G}$ binding model [17].

\section{Non-polar surface is a minor feature in $\Delta \Delta G$ binding models (minimal sidechain repacking, Minimal-HighSA)}

Empirical binding models generally target either complexes or differences between wild-type and mutant com- 
Table 2: Weights for ASA-based binding models.

\begin{tabular}{|c|c|c|c|c|c|c|c|c|c|c|}
\hline Model type & $\Delta \mathbf{G}$ & $\Delta \mathbf{G}$ & $\Delta \mathbf{G}$ & $\Delta \mathbf{G}$ & $\Delta \Delta \mathbf{G}$ & $\Delta \Delta \mathbf{G}$ & $\Delta \mathbf{G}$ & $\Delta \mathbf{G}$ & $\Delta \Delta \mathbf{G}$ & $\Delta \Delta \mathbf{G}$ \\
\hline$\Delta \mathbf{G}_{\text {ROT-TRANS }}$ & 0 & 10 & 0 & 10 & - & - & 0 & 0 & - & - \\
\hline Un/Locked & $\mathrm{L}$ & $\mathrm{L}$ & $U$ & $U$ & $\mathrm{~L}$ & $U$ & $\mathrm{~L}$ & $U$ & L & $U$ \\
\hline Packing Scheme & \multicolumn{6}{|c|}{ Minimal-HighSA } & \multicolumn{4}{|c|}{ Complete-LowSA } \\
\hline ASA-NP & 0.73 & 0.79 & 0.60 & 0.65 & 0.07 & -0.04 & 0.58 & 0.56 & -0.31 & -0.23 \\
\hline ASA-P & -0.04 & 0.04 & -0.08 & 0.02 & -0.73 & -0.67 & -0.05 & -0.11 & -0.65 & 0.04 \\
\hline IONIS-FDDH & -0.01 & -0.04 & 0.15 & 0.13 & 0.21 & 0.08 & 0.10 & 0.10 & 0.17 & 0.16 \\
\hline IONIS-DESOLV & 0.15 & 0.09 & 0.06 & 0.01 & 1.29 & 0.97 & 0.20 & 0.19 & 0.67 & 0.61 \\
\hline SC-ROT & 0.12 & 0.10 & -0.22 & -0.30 & -0.02 & -1.61 & 0.03 & -0.02 & -0.50 & -0.61 \\
\hline $\mathbf{R}^{2}$ correlation & 0.69 & 0.64 & 0.61 & 0.58 & 0.61 & 0.64 & 0.69 & 0.68 & 0.54 & 0.55 \\
\hline Figure/Panel & la & - & Id & - & $2 a$ & $2 b$ & 6 & - & - & - \\
\hline
\end{tabular}

Weights are either $w$ for $\Delta G$ models or $w^{\prime}$ for $\Delta \Delta G$ models. $U / L$ is Unlocked/Locked interfacial sidechains, applied to SC-ROT.

plexes, but not both. We are interested in whether $\Delta \mathrm{G}$ and $\Delta \Delta \mathrm{G}$ models show the same relative importance of model features. Figure 2 shows results for $\Delta \Delta \mathrm{G}$ models, where the rotation/translation term cancels between wild-type and mutant systems. Again reasonable fits are obtained, although this time the Unlocked interfacial sidechain entropy term gives a slightly better model than the locked term (compare panels (a) and (b) of Figure 2). As for the wild-type calculations, these two terms do not correlate well (not shown). A major difference to $\Delta \mathrm{G}$ models (Table 2 ) is that non-polar surface area has a small weight, and a poor correlation with the measured binding data (Table 4). The $\Delta \Delta \mathrm{G}_{\text {ASA-P, }}, \Delta \Delta \mathrm{G}_{\text {IONIS-DESOLV }}$ and $\Delta \Delta \mathrm{G}_{\mathrm{SC}-\mathrm{ROT}}$ terms correlate to such a degree that individually they can adopt a large and unphysical weighting, with compensation by the other features.

\section{A $\Delta G$ binding model fits poorly to measured $\Delta \Delta G$ (minimal sidechain repacking, Minimal-HighSA)}

Figure 3 demonstrates discrepancy between $\Delta \mathrm{G}$ and $\Delta \Delta \mathrm{G}$ models. The $\Delta \mathrm{G}$ model of Figure 1 (a) (see also Table 2) has been applied to the mutant complexes, and compared with measured $\Delta \mathrm{G}$. Within clusters (representing the mutant sets), the spreads of calculated $\Delta \mathrm{G}$ are too small to reproduce variation in the measurements. This issue of model incompatibility is not resolved when a single $\Delta \mathrm{G}$ model is fit to $\Delta \mathrm{G}$ data for wild-type and mutant complexes (not shown). Some resolution of the discrepancy could be achieved if assessment of buried non-polar area were inaccurate in $\Delta \mathrm{G}$ or $\Delta \Delta \mathrm{G}$ models, and/or if the strategies for mutant selection, and perhaps the systems undergoing mutagenesis, emphasise other features over non-polar properties. The second factor is unlikely to be the whole story, since polar features are down-weighted in the $\Delta \mathrm{G}$ models. With regard to the first factor, the meanfield packing algorithm has been modified such that sidechain rotamers can be chosen that will tend towards maximal packing with the rest of a protein (LowSA).

\section{Repacking sidechains with the mean-field algorithm}

Figure 4 shows the scheme for generation of mutant structures, using the various sidechain packing schemes that are available, Minimal or Complete and HighSA or LowSA. The previous Results sections referred to MinimalHighSA. Following the observation that buried non-polar ASA dominates $\Delta \mathrm{G}$, but not $\Delta \Delta \mathrm{G}$ (with Minimal-HighSA binding models), we now consider the effect of using Complete-LowSA repacking. We theorise that in models of mutant structures, particularly those with replacement by alanine, sidechains will relax into the space left by the mutation. Since there may be different constraints on

Table 3: Correlations (R) for $\Delta \mathbf{G}$ model features in wild-type complexes, Minimal-HighSA packing

\begin{tabular}{|c|c|c|c|c|c|c|c|}
\hline & $\triangle$ ASA-NP & $\triangle$ ASA-P & $\Delta$ IONIS-FDDH & $\triangle I O N I S-D E S O L V$ & $\Delta$ SC-ROT Locked & $\Delta$ SC-ROT Unlocked & $\Delta \mathbf{G}_{\text {EXP }}$ \\
\hline$\triangle$ ASA-NP & I & & & & & & \\
\hline$\triangle$ ASA-P & 0.57 & I & & & & & \\
\hline$\Delta$ IONIS-FDDH & 0.31 & 0.50 & I & & & & \\
\hline$\triangle I O N I S-D E S O L V$ & 0.55 & 0.92 & 0.51 & I & & & \\
\hline$\Delta$ SC-ROT L & -0.46 & -0.69 & -0.25 & -0.76 & I & & \\
\hline$\Delta$ SC-ROT U & -0.39 & -0.14 & 0.13 & -0.24 & 0.35 & I & \\
\hline$\Delta \mathbf{G}_{\mathrm{EXP}}$ & 0.74 & 0.45 & 0.42 & 0.54 & -0.17 & -0.31 & I \\
\hline
\end{tabular}


(a)

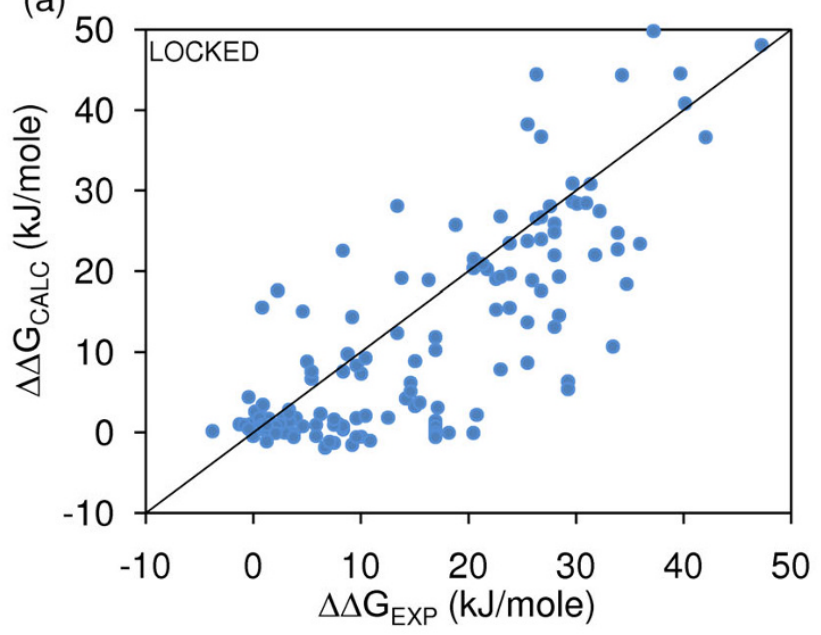

(b)

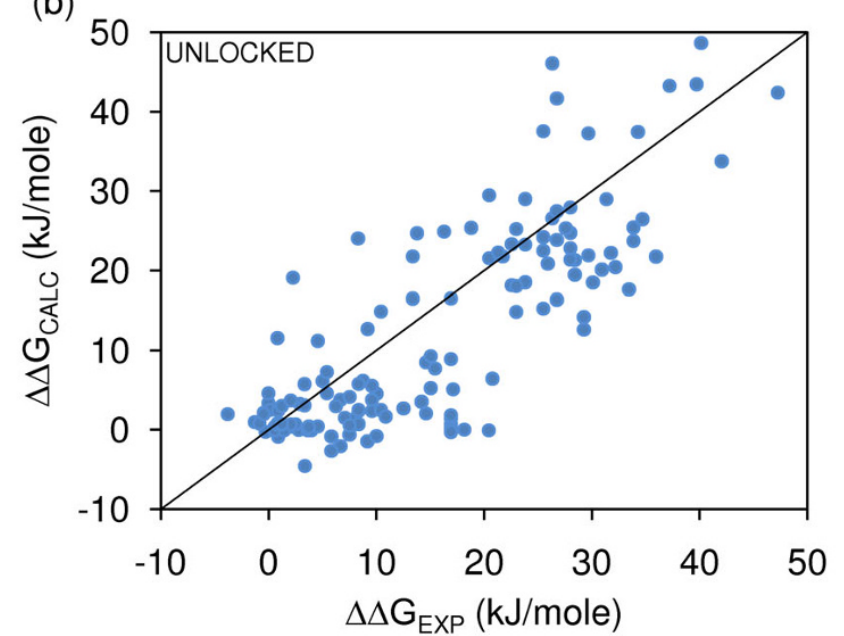

Figure 2

$\Delta \Delta \mathbf{G}$ models for Locked and Unlocked interfacial sidechains, Minimal-HighSA. Binding models fit to $\Delta \Delta G_{\mathrm{EXP}}$, for Minimal-HighSA repacking. (a) $\Delta \mathrm{G}_{\mathrm{SC}-\mathrm{ROT}}$ calculated for Locked interfacial sidechains $\left(\mathrm{R}^{2}=0.6 \mathrm{I}\right)$. (b) $\Delta \mathrm{G}_{\mathrm{SC}-\mathrm{ROT}}$ calculated for Unlocked sidechains $\left(\mathrm{R}^{2}=0.64\right)$.

such relaxation in the complexed and uncomplexed states, this could lead to changes in buried area.

The potential for LowSA prediction is shown with the wild-type complexes in Figure 5(a), where the total ASA values are shown for: experimental structures, CompleteHighSA repacking and Complete-LowSA repacking. LowSA gives an overall result more in accord with experiment. Figure 5(b) shows an example for the barnase-barstar complex (1b27, [26]), where more sidechains packed with Complete-HighSA protrude from the crystal structure molecular surface, than do those packed with Complete-LowSA. In terms of predicted buried ASAs (polar and non-polar combined), Figure 5(c) shows that for the set of mutants used in this study, Complete-LowSA repacking gives consistently more burial than does Complete-HighSA. This result holds when mutant systems are differenced to wild-type, and also whether the mutants are to alanine or other amino acids (not shown). Therefore the $\Delta \Delta \mathrm{G}_{\text {ASA-NP }}$ and $\Delta \Delta \mathrm{G}_{\text {ASA-P }}$ terms are larger (for a given weighting) with Complete-LowSA packing than with Complete-HighSA, and we next examined whether this alters the discrepancy between $\Delta \mathrm{G}$ and $\Delta \Delta \mathrm{G}$ models.

\section{Combining $\triangle G$ and $\triangle \Delta G$ binding models (Complete-LowSA sidechain repacking)}

Table 2 lists the best fit models for Complete-LowSA packing applied to the wild-type complexes. The "Complete" packing schemes allow for sidechain relaxation between complexed and uncomplexed forms, including sidechain repacking in the experimentally-determined structure of the complex. Generally sidechains away from the interface will pack similarly in the Complete-LowSA scheme in complexed and uncomplexed forms, therefore cancelling in the binding calculations. The $\Delta \mathrm{G}$ models for CompleteLowSA are not very different from those for MinimalHighSA. Models using Locked or Unlocked interfacial sidechains for the rotameric entropy estimation are

Table 4: Correlations (R) for $\Delta \Delta$ G model features (mutant and wild-type complexes differenced), Minimal-HighSA packing

\begin{tabular}{|c|c|c|c|c|c|c|c|}
\hline & $\Delta \Delta$ ASA-NP & $\Delta \Delta$ ASA-P & $\Delta \Delta$ IONIS-FDDH & $\begin{array}{l}\triangle \Delta \text { IONIS- } \\
\text { DESOLV }\end{array}$ & $\begin{array}{l}\Delta \Delta \text { SC-ROT } \\
\text { Locked }\end{array}$ & $\begin{array}{l}\Delta \Delta \text { SC-ROT } \\
\text { Unlocked }\end{array}$ & $\Delta \Delta \mathbf{G}_{\mathrm{EXP}}$ \\
\hline$\Delta \Delta$ ASA-NP & 1 & & & & & & \\
\hline$\Delta \Delta$ ASA-P & -0.13 & I & & & & & \\
\hline$\Delta \Delta$ IONIS-FDDH & -0.06 & 0.11 & 1 & & & & \\
\hline $\begin{array}{l}\triangle \Delta \text { IONIS- } \\
\text { DESOLV }\end{array}$ & -0.22 & 0.87 & 0.11 & I & & & \\
\hline$\Delta \Delta$ SC-ROT L & -0.26 & 0.07 & 0.01 & 0.13 & I & & \\
\hline$\Delta \Delta$ SC-ROT U & 0.08 & -0.52 & -0.35 & -0.62 & -0.11 & $\mathrm{I}$ & \\
\hline$\Delta \Delta \mathbf{G}_{\text {EXP }}$ & -0.18 & 0.55 & 0.30 & 0.74 & 0.29 & -0.65 & I \\
\hline
\end{tabular}




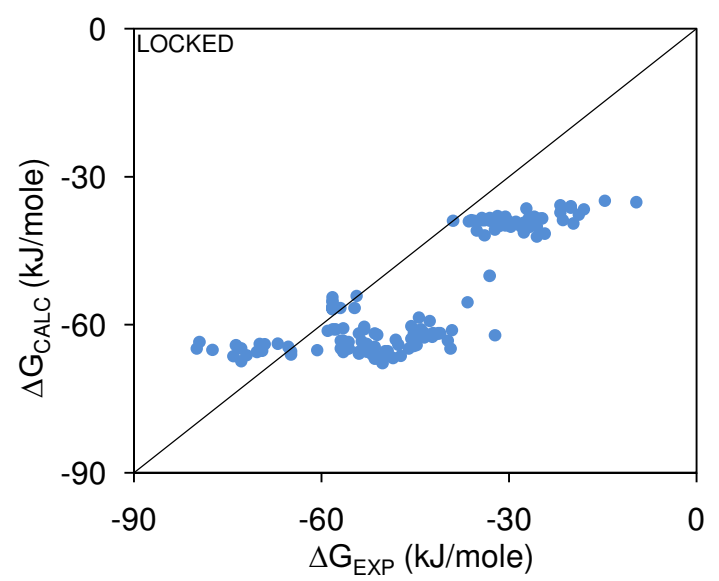

Figure 3

Wild-type complexes $\Delta \mathbf{G}$ model applied to mutant complexes, Minimal-HighSA. The $\Delta \mathrm{G}$ model fit to wildtype complexes, with Locked interfacial sidechains and $\Delta \mathrm{G}_{\text {ROT-TRANS }}=0$ applied to mutant complexes, all calculations with Minimal-HighSA packing $\left(R^{2}=0.66\right)$.

almost identical for Complete-LowSA. Also, the differences between $\Delta \mathrm{G}_{\text {ROT-TRANS }}=0$ and $\Delta \mathrm{G}_{\text {ROT-TRANS }}=10$ calculations are again small (not shown).

Figure 6 shows the best-fit Complete-LowSA $\Delta \mathrm{G}$ binding model, derived for wild-type complexes, applied to $\Delta \mathrm{G}$ data for mutant complexes (with all molecules repacked in the Complete-LowSA scheme). This is for rotameric entropy estimated from Locked sidechains at the interface, although the low weighting in the model means this will have little influence, and with $\Delta \mathrm{G}_{\text {ROT-TRANS }}=0$. Figure 6 can be compared directly with Figure 3 (the equivalent Minimal-HighSA calculation), and it can be seen that these plots are qualitatively similar. We had hypothesised that the increase in buried non-polar surface area for Complete-LowSA over Minimal-HighSA models, could lead to greater consistency between $\Delta \mathrm{G}$ and $\Delta \Delta \mathrm{G}$ models. This is not the case.

\section{Recalculating ASA at lower solvent probe radius}

Since $\Delta \mathrm{G}_{\mathrm{ASA}-\mathrm{NP}}$ drives agreement for $\Delta \mathrm{G}$ models, but not for $\Delta \Delta \mathrm{G}$ models (whichever sidechain packing is used), we looked more closely at this property in the mutant interfaces. From Figure 7(a), (b) it is apparent that both Minimal-HighSA and Complete-LowSA schemes give a spread of $\Delta \Delta$ (ASA-NP) values around zero, albeit with larger magnitude in the Complete-LowSA scheme. Since $\Delta \Delta \mathrm{G}_{\mathrm{EXP}}$ is almost exclusively of one sign, it is clear that $\Delta \Delta \mathrm{G}_{\mathrm{ASA}-\mathrm{NP}}$ will be down-weighted in a best fit linear model. Figure 7 (c) shows that the dual sign spread of $\Delta \Delta$ (ASA-NP) is largely negated when the solvent probe radius is reduced from $1.4 \AA$ to $0.5 \AA$, but the correlation with $\Delta \Delta \mathrm{G}_{\mathrm{EXP}}$ remains poor. These results indicate that $\Delta \Delta \mathrm{G}_{\mathrm{ASA}-\mathrm{NP}}$, with the current repacking schemes, is not an effective feature with which to understand $\Delta \Delta \mathrm{G}$ binding models. Visual inspection (not shown) reveals that relatively large non-polar surfaces may be revealed upon mutation, and whether or not these are solvent accessible depends on the fine detail of packing differences between a complex and its components. Whereas ASA-NP is the most prominent feature in $\Delta \mathrm{G}$ models, either it is not an appropriate description for interfacial relaxation in response to mutation, or current modelling of interfacial relaxation is insufficient.

\section{Volume-based solvation}

We reasoned that a volume-based solvation function would be less sensitive to the details of packing changes than a solvent accessible area-based feature. This is consistent with the more prominent role played by $\Delta \mathrm{G}_{\mathrm{IONIS}}$ DESOLV in $\Delta \Delta \mathrm{G}$ models, as opposed to $\Delta \mathrm{G}$ models. Solvation shell models have been investigated previously in the context of solute/protein structure and stabilisation [2729]. Here we develop simple properties to replace $\Delta /$ $\Delta \Delta \mathrm{G}_{\mathrm{ASA}-\mathrm{NP}}$ and $\Delta / \Delta \Delta \mathrm{G}_{\mathrm{ASA}-\mathrm{P}}$ in the binding models, in which solvation shell volumes are calculated for each atom, on a grid. The volumes cover a shell of $2.8 \AA$ thickness around atomic van der Waals radii, and are assigned polar or non-polar according to atom type. Then $\Delta$ (complexation) and $\Delta \Delta$ (mutant to wild-type and complexation differences) are calculated for $\mathrm{G}_{\mathrm{VOL}-\mathrm{NP}}$ and $\mathrm{G}_{\mathrm{VOL}-\mathrm{P}}$. This model affords a rapid calculation, and volume-based features give the uniform sign behaviour expected for mutants that generally involve reduction of sidechain size (not shown), unlike the distribution of $\Delta \Delta$ (ASA-NP) (Figure $7(\mathrm{a}))$.

Figure 8 compares Minimal-HighSA models for area and volume-based features (Table 5), where models have been fit to wild-type $\Delta \mathrm{G}$ data and calculated and plotted for the mutant $\Delta \mathrm{Gs}$. The mutant data in both panels show an unequal distribution around the $\Delta \mathrm{G}_{\mathrm{CALC}}=\Delta \mathrm{G}_{\mathrm{EXP}}$ line, since the 4 underlying systems are a subset of the 20 wildtype complexes used to generate the area and volumebased $\Delta \mathrm{G}_{\mathrm{CALC}}$ models. A relatively flat spread of mutant clusters for the area-based model is much less apparent with the volume-based model, indicating a more consistent modelling of variation within these clusters, although $\mathrm{R}^{2}$ is not much different between the two panels of Figure 8 . Further evidence of the effectiveness of volume-based modelling is seen when comparing equivalent $\Delta G$ and $\Delta \Delta \mathrm{G}$ models in Tables 2 and 5. For Minimal-HighSA and Locked interfacial sidechains, feature weights change entirely between ASA-based $\Delta \mathrm{G}$ and $\Delta \Delta \mathrm{G}$ models (e.g. with the non-polar ASA term going from dominating to being insignificant). For volume-based models, it is actually the polar term that is more important, but this is 


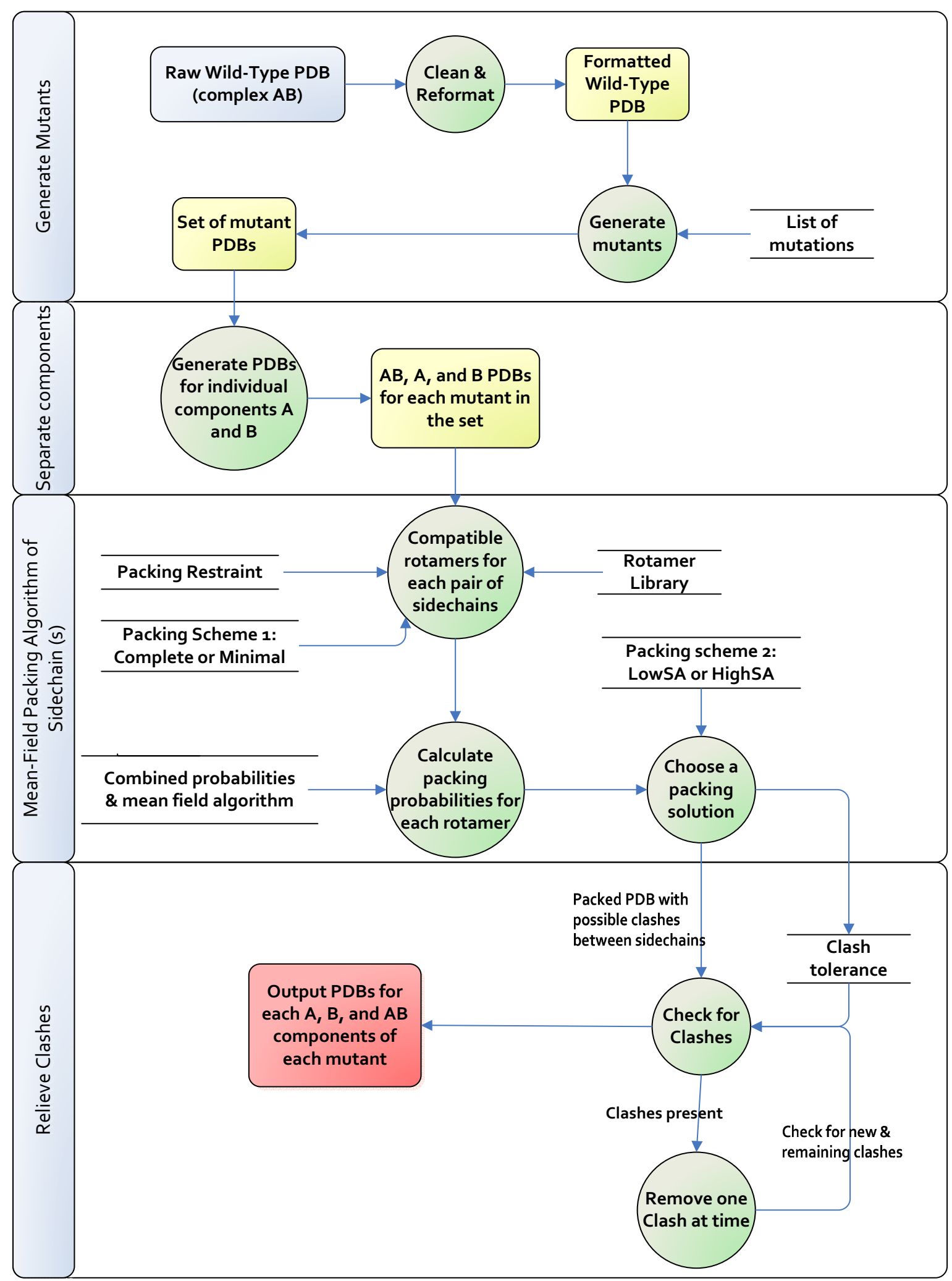

Figure 4

Scheme for generation of mutant complexes and different sidechain packing. 


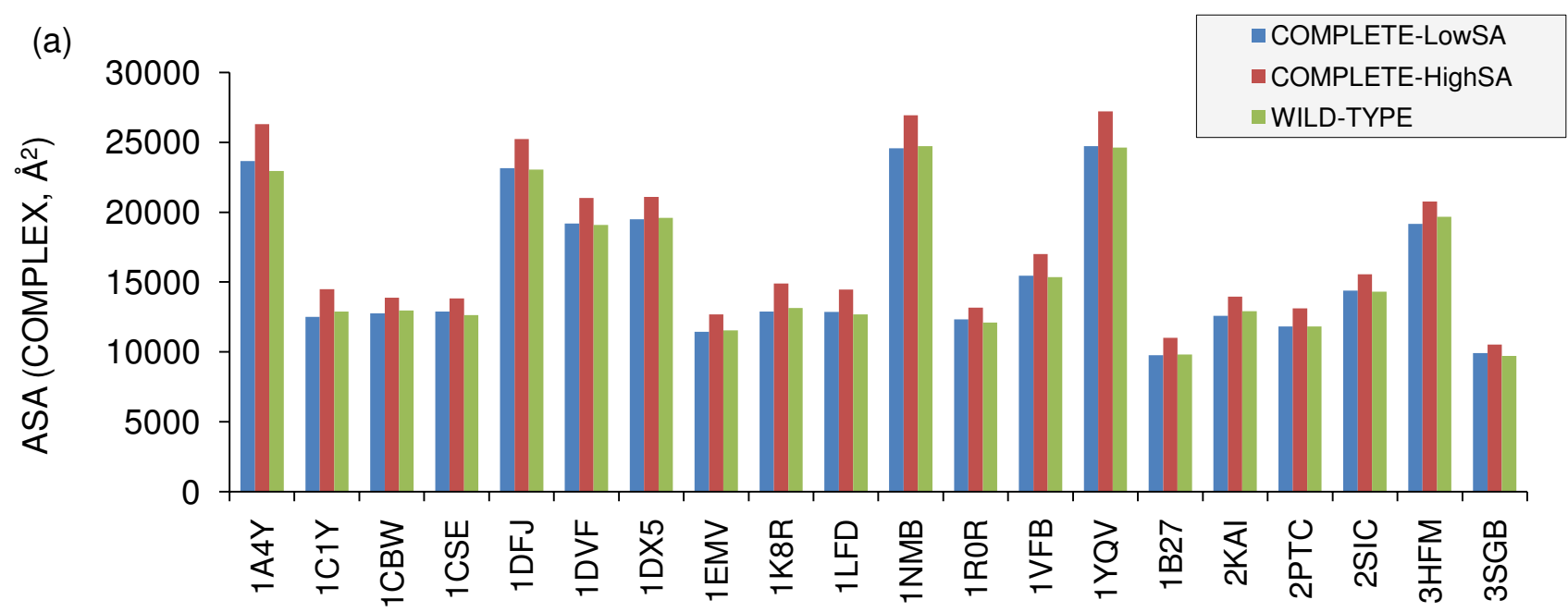

(b)

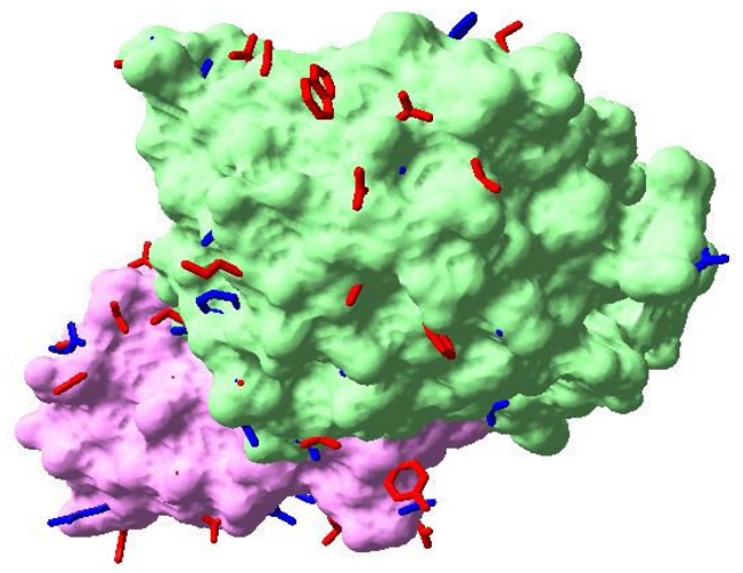

(c)

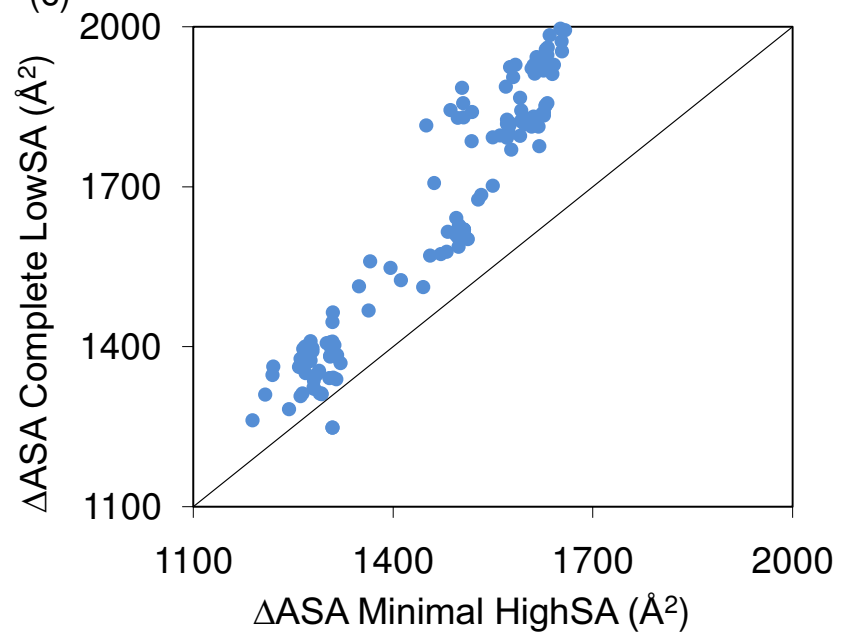

Figure 5

Complete-HighSA and Complete-LowSA sidechain packing compared. (a) Total ASA for wild-type complexes, calculated for the experimental structures (wild-type), and repacking of all sidechains in the Complete-HighSA and CompleteLowSA schemes. (b) Molecular surface of experimental structure (I b27 subunits A and D) for barnase (green) - barstar (pink), with Complete-HighSA (red) and Complete-LowSA (blue) repacked sidechains protruding. Drawn with the program SwissPdbViewer [48]. (c) Correlation between $\triangle($ ASA) (total buried surface area) in the complexes for Complete-HighSA and Complete-LowSA repacking $\left(R^{2}=0.91\right)$.

maintained on moving from $\Delta \mathrm{G}$ to $\Delta \Delta \mathrm{G}$. Weight variations for $\Delta \mathrm{G}$ and $\Delta \Delta \mathrm{G}$ models with Complete-LowSA repacking are more complex.

Looking at calculations with Minimal-HighSA repacking, it appears that even simple volume calculations are capturing common properties in the $\Delta \mathrm{G}$ and $\Delta \Delta \mathrm{G}$ processes, a behaviour that has largely defeated the ASA-based calculations. The volume-based terms are particularly rapid to calculate, and could provide the basis for simple, low resolution, computational screens of interface viability.
Table 6 shows $\Delta \mathrm{G}$ and $\Delta \Delta \mathrm{G}$ models derived using only two volume-based solvation terms. The $\mathrm{R}^{2}$ values for Minimal-HighSA models with just two features are close to those of the best fits for the more extensive MinimalHighSA models given in Table 5.

Our investigation of simple, empirical models for binding that can generalise between $\Delta \mathrm{G}$ and $\Delta \Delta \mathrm{G}$ applications, contrasts with interface-specific methods using quantitative structure-activity relationships that highlight residues of particular importance [31]. In practice both approaches 


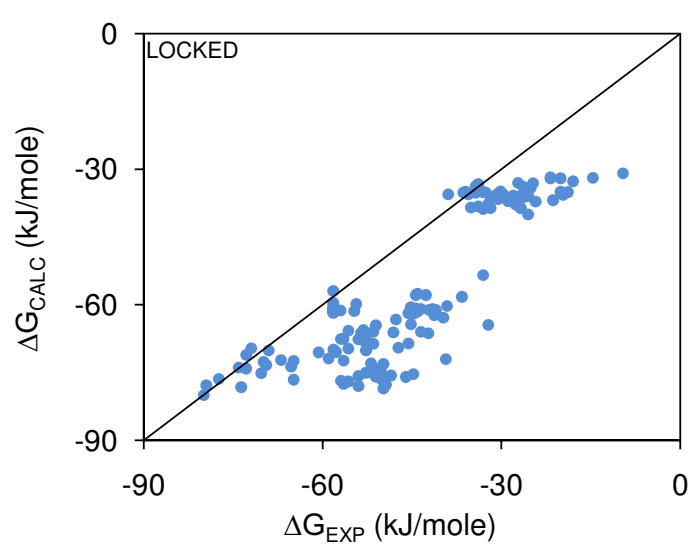

\section{Figure 6}

Wild-type complexes $\Delta \mathbf{G}$ model applied to mutant complexes, Complete-LowSA. The $\Delta \mathrm{G}$ model fit to wild-type complexes, with Locked interfacial sidechains and $\Delta \mathrm{G}_{\text {ROT-TRANS }}=0$ applied to mutant complexes, all calculations with Complete-LowSA packing $\left(R^{2}=0.73\right)$.

have the same overall aim, i.e. predictive capability for protein-protein interactions; and the same constraints, with sets of correlated features (Tables 3 and 4) and an imprecise understanding of the underlying conformational and energetic framework. Our observation that volume-based terms perform better than area-based terms in $\Delta \Delta \mathrm{G}$ models highlights this latter problem. It is possible that modified area-based terms, for example with conformational sampling and weighting, could improve performance.

\section{Conclusion}

We find that binding models, using minimal sidechain repacking and ASA-based solvation terms, are quite different depending on whether they are fit to data for $\Delta G$ (wild-type complexes) or $\Delta \Delta \mathrm{G}$ (modelled mutant complexes differenced to wild-type complexes). Whereas buried non-polar area dominates the $\Delta \mathrm{G}$ model (Figure 1, Table 2), consistent with previous work [17], other interactions assume much greater importance for the mutant complexes (Figure 2, Table 2), and the $\Delta \mathrm{G}$ binding model does not reproduce the spread of $\Delta \mathrm{G}_{\mathrm{EXP}}$ within mutant sets (Figure 3).

Investigating whether different sidechain repacking could alter this discrepancy, a scheme for packing sidechains towards protein structure has been derived from a meanfield framework (Figure 4). This method, which we label Complete-LowSA since all sidechains (mutated or not) are repacked, is promising in terms of a better agreement with total ASA for experimental complexes, and in giving larger buried surface areas upon complexation than the Minimal-HighSA scheme (Figure 5). However, this does not lead to significant increase in the importance of nonpolar buried area in best fit $\Delta \Delta \mathrm{G}$ models, and there remains a discrepancy between $\Delta \mathrm{G}$ and $\Delta \Delta \mathrm{G}$ models (Figure 6). Further analysis of $\Delta \Delta$ (ASA-NP) revealed a spread around zero that mitigates against fitting to $\Delta \Delta \mathrm{G}_{\mathrm{EXP}}$ which is predominantly single sign (Figure 7 ). These results indicate that either non-polar buried area is not important for $\Delta \Delta \mathrm{G}$ modelling, which would be surprising given its role in $\Delta \mathrm{G}$ modelling, or that we are not capturing the complexity of sidechain (and potentially mainchain) conformational rearrangement upon mutation.

Of the features studied, ionisable group-based electrostatics contributes relatively little in best fits to experimental data, for both $\Delta \mathrm{G}$ and $\Delta \Delta \mathrm{G}$ models. Clearly there will be instances where ionisable groups will contribute substantially to interfacial energetics, and mediate processes such as a physiological pH-dependence of binding. In general though, studies of optimal predictors of interfacial propensity show that net charge is (in relative terms) excluded, part of an overall tendency for ease of desolvation at interfaces [30]. For most $\Delta \mathrm{G}$ and $\Delta \Delta \mathrm{G}$ models, sidechain rotameric entropy plays a relatively small part (Table 2), and where the weight is large may be partly due to correlations with other properties (Tables 3, 4). In $\Delta \Delta \mathrm{G}$ models with ASA-based solvation, $\Delta \Delta \mathrm{G}_{\text {ASA-P }}$ and $\Delta \Delta \mathrm{G}_{\text {IONIS- }}$ DESOLV assume more importance. Polar solvation may be reflecting overall burial change in the interface upon mutation, rather than indicative of particular favourable polar interactions. The effects of mutations on polar area relative to non-polar area are somewhat different. The polar area equivalent to the non-polar area data plotted in Figure 7 (a) (Minimal-HighSA) is qualitatively similar, whereas the polar area equivalent to Figure 7(b) (Complete-LowSA) is qualitatively different, being largely of a single sign (not shown).

Empirical desolvation for ionisable groups, describing an estimate of the entropy of water molecule liberation, is a simple volume-based term. We therefore tested the capacity for volume-based terms in general to account for solvation changes in $\Delta \Delta \mathrm{G}$ models, in place of area-based terms. The volume solvation features assume importance in all models, reducing the discrepancy between $\Delta \mathrm{G}$ and $\Delta \Delta \mathrm{G}$ models (Figure 8). Volume-based models are part of the molecular mechanics force-fields in some applications $[20,29]$, and $\Delta \Delta G$ models with volume-based solvation terms have been used, in part for faster calculation $[19,20]$. In the current work, we show that even a very simple solvation shell model is effective in improving consistency of $\Delta \mathrm{G}$ and $\Delta \Delta \mathrm{G}$ models. Surface area-based modelling for $\Delta \Delta \mathrm{G}$ fails since ASA is particularly sensitive to relatively small conformational changes, even for single site mutations. In contrast volume based models are less sensitive, and a simple binding model for $\Delta \mathrm{G}$ and $\Delta \Delta \mathrm{G}$ 

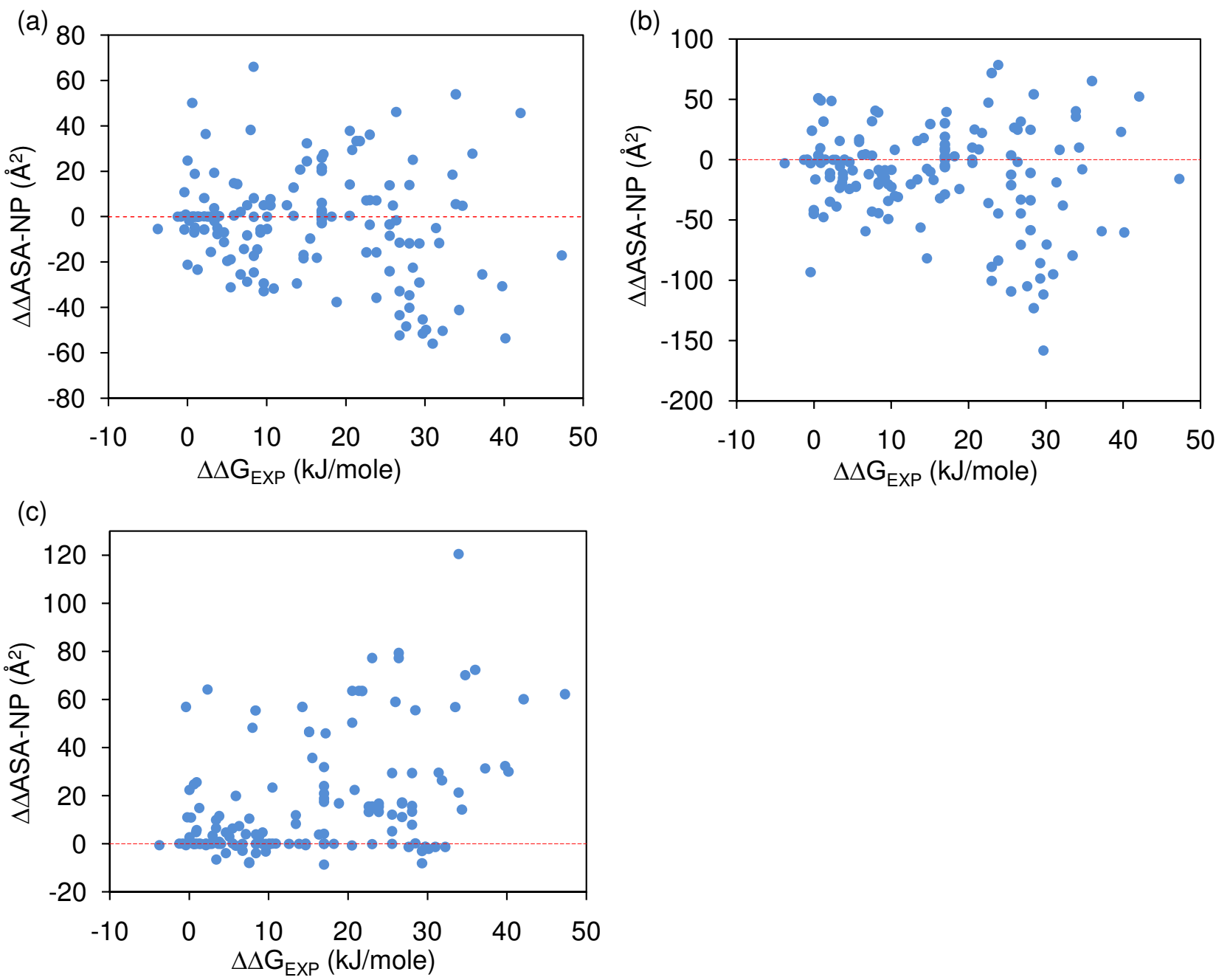

\section{Figure 7}

$\Delta \Delta$ (ASA-NP) does not correlate with $\Delta \Delta \mathbf{G}_{\mathrm{EXP}}$, and is of variable sign. (a) $\Delta \Delta$ (ASA-NP) calculated with Minimal-HighSA packing does not correlate with $\Delta \Delta G_{E X P}\left(R^{2}=0.00\right)$, and can be positive or negative (red line drawn at $\left.\Delta \Delta(A S A-N P)=0\right)$. (b) Qualitatively similar behaviour to that for panel (a) is seen for Complete-LowSA repacking $\left(R^{2}=0.02\right)$. (c) Reducing the solvent probe radius from I.4 $\AA$ to $0.5 \AA$ gives mostly uniform sign of $\Delta \Delta(A S A-N P)$, but still poor correlation with $\Delta \Delta G_{E X P}\left(R^{2}=0.18\right)$.

can be constructed with just polar and non-polar volumebased features. This could be useful in computational assessment of the validity of comparative modelled interfaces, and our simple implementation is made available for such analyses.

\section{Methods}

\section{Binding data and protein structures}

Protein complexes were selected where the literature and structural databases give no evidence of major conformational change in the mainchain upon complexation, and for which measured $\Delta \mathrm{G}$ is available. The literature e.g. [31] and various databases were used to search for binding energies associated with complexation, the alanine scanning energetics database (ASEdb, [32]), the protein-protein interactions thermodynamic database (PINT, [33]), and the ProTherm thermodynamic database for proteins and mutants [34]. Imposition of limited conformational change led to the inclusion of just 20 complexes, which are listed in Table 1 with their measured binding energies. In cases where binding data are recorded for multiple conditions, that most closely corresponding to our calculation conditions of $300 \mathrm{~K}, 0.15 \mathrm{M}$ ionic strength, $\mathrm{pH} \mathrm{7,}$ were chosen. The data in Table 1 were used to construct $\Delta \mathrm{G}$ binding models. In calculations, only structure files (from the PDB, [4]) for the complex were used. All components (free proteins and mutated proteins) were derived from these coordinates.

For mutant data in $\Delta \Delta \mathrm{G}$ model fitting, 4 of the systems used in $\Delta \mathrm{G}$ modelling were included. The restriction of no major mainchain changes was therefore carried over to 

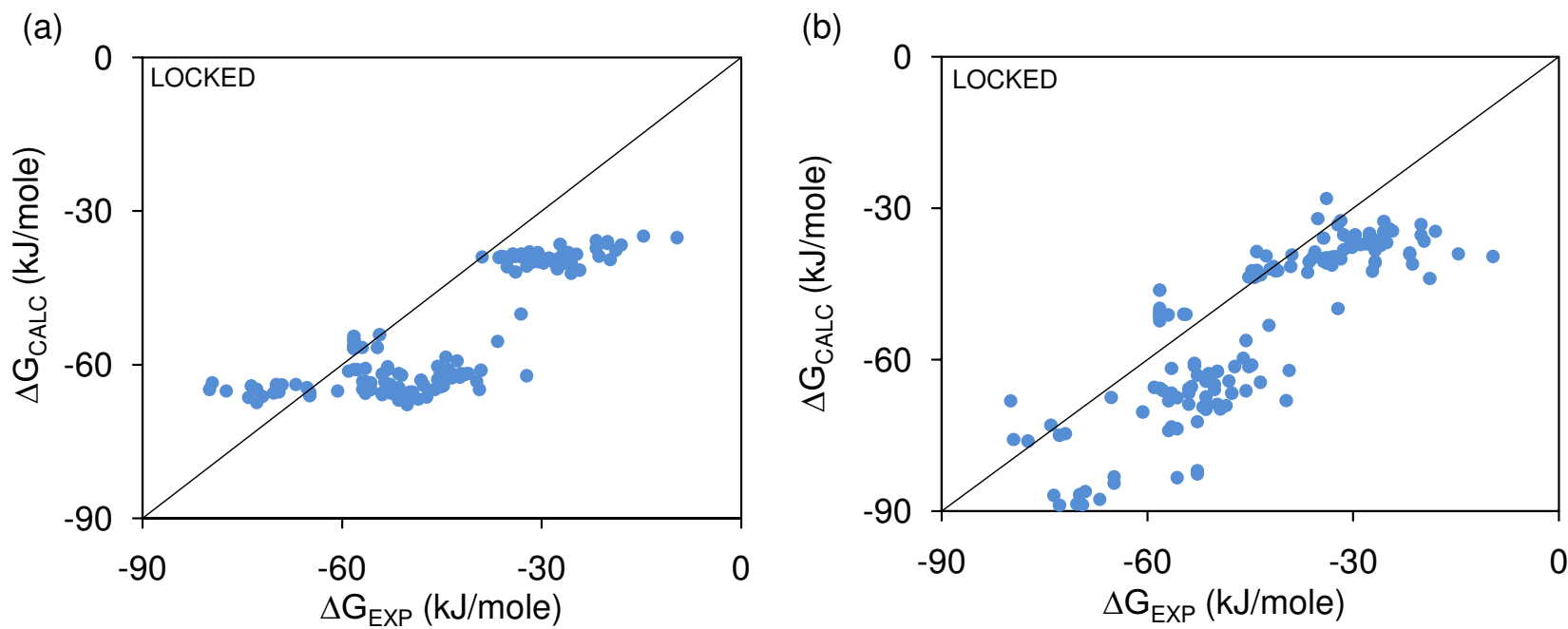

\section{Figure 8}

ASA-based and volume-based solvation $\Delta \mathbf{G}$ models compared. Calculations for $\Delta \mathrm{G}$ of mutant complexes, using $\Delta \mathrm{G}$ models fit to wild-type complex data, with $\Delta \mathrm{G}_{\mathrm{SC}-\mathrm{ROT}}$ calculated for Locked interfacial sidechains, Minimal-HighSA repacking and $\Delta G_{\text {ROT-TRANS }}=0$. (a) ASA-based polar and non-polar solvation terms $\left(R^{2}=0.66\right)$. (b) Volume-based solvation $\left(R^{2}=0.68\right)$. Although there is little difference in $R^{2}$, the spread in $\Delta G_{C A L C}$ within mutant systems (i.e. based on the same wild-type) is larger with volume calculations.

the mutants $\Delta \Delta \mathrm{G}$ analysis. These systems, and the number of mutants associated with each, are: barnase-barstar, 64 mutants; chymotrypsin-BPTI, 15 mutants; ralGDS-ras, 49 mutants; trypsin-BPTI, 15 mutants. Where mutants are solely changes to alanine, no repacking is required in the Minimal-HighSA or Minimal-LowSA schemes. CompleteHighSA and Complete-LowSA schemes will repack all sidechains.

\section{Binding model}

We employed an empirical binding model with contributions from the rigid body rotational and translational entropy of complexation ( $\left.\Delta \mathrm{G}_{\text {ROT-TRANS }}\right)$, buried non-polar surface area $\left(\Delta \mathrm{G}_{\mathrm{ASA}-\mathrm{NP}}\right)$, buried polar surface area $\left(\Delta \mathrm{G}_{\mathrm{ASA}}\right.$ $\left.{ }_{\mathrm{P}}\right)$, ionisable group charge interactions $\left(\Delta \mathrm{G}_{\mathrm{IONIS-FDDH}}\right), \mathrm{a}$ term to approximate the free energy of water molecule liberation upon ionisable group burial ( $\left.\Delta \mathrm{G}_{\mathrm{IONIS}-\mathrm{DESOLV}}\right)$, and sidechain rotameric entropy $\left(\Delta \mathrm{G}_{\mathrm{SC}-\mathrm{ROT}}\right)$. Thus;

$$
\begin{gathered}
\Delta \mathrm{G}_{\text {CALC }}=\Delta \mathrm{G}_{\text {ROT-TRANS }}+\mathrm{w}_{\text {ASA-NP }} \Delta \mathrm{G}_{\text {ASA-NP }}+\mathrm{w}_{\text {ASA-P }} \Delta \mathrm{G}_{\text {ASA-P }}+ \\
\mathrm{w}_{\text {IONIS-FDDH }} \Delta \mathrm{G}_{\text {IONIS-FDDH }}+\mathrm{w}_{\text {IONIS-DESOLV }} \Delta \mathrm{G}_{\text {IONIS-DESOLV }}+ \\
\mathrm{w}_{\text {SC-ROT }} \Delta \mathrm{G}_{\text {SC-ROT }}
\end{gathered}
$$

where the various pre-multipliers (w) are the weights to be adjusted in model fitting to experimental data. More detail follows for the individual terms.

The rotational and translational entropy change associated with complexation of two rigid bodies has been stud-

\begin{tabular}{|c|c|c|c|c|c|c|c|c|}
\hline Model type & $\Delta \mathbf{G}$ & $\Delta \mathbf{G}$ & $\Delta \Delta \mathbf{G}$ & $\Delta \Delta \mathbf{G}$ & $\Delta \mathbf{G}$ & $\Delta \mathbf{G}$ & $\Delta \Delta \mathbf{G}$ & $\Delta \Delta \mathbf{G}$ \\
\hline$\Delta \mathbf{G}_{\text {ROT-TRANS }}$ & 0 & 0 & - & - & 0 & 0 & - & - \\
\hline Un/Locked & $\mathrm{L}$ & $\mathrm{U}$ & $\mathrm{L}$ & $\mathrm{U}$ & $\mathrm{L}$ & $\mathrm{U}$ & $\mathrm{L}$ & $\mathrm{U}$ \\
\hline Packing Scheme & \multicolumn{4}{|c|}{ Minimal-HighSA } & \multicolumn{4}{|c|}{ Complete-LowSA } \\
\hline VOL-NP & -0.12 & -0.11 & -0.31 & -0.28 & 0.20 & 0.24 & 0.04 & 0.02 \\
\hline VOL-P & 1.00 & 0.85 & 1.79 & 1.45 & -0.03 & -0.08 & 0.55 & 0.61 \\
\hline IONIS-FDDH & 0.44 & 0.43 & -0.01 & -0.05 & 0.19 & 0.18 & 0.28 & 0.29 \\
\hline IONIS-DESOLV & -0.19 & 0.11 & 0.26 & 0.23 & 0.17 & 0.26 & 0.30 & 0.35 \\
\hline SC-ROT & -0.07 & -0.49 & -0.01 & -1.04 & -0.06 & 0.08 & -0.24 & -0.29 \\
\hline $\mathbf{R}^{2}$ correlation & 0.46 & 0.50 & 0.62 & 0.64 & 0.64 & 0.66 & 0.64 & 0.66 \\
\hline
\end{tabular}
ied experimentally and theoretically $[25,35,36]$.

Table 5: Weights for volume-based binding models.

Weights are $w$ for $\Delta \mathrm{G}$ model, $\mathrm{w}^{\prime}$ for $\Delta \Delta \mathrm{G}$ models. $\mathrm{U} / \mathrm{L}$ is Unlocked/Locked interfacial sidechains, applied to SC-ROT. 
Table 6: Weights for binding models with only volume-based features.

\begin{tabular}{|c|c|c|c|c|}
\hline Model type & $\Delta \mathbf{G}$ & $\Delta \Delta \mathbf{G}$ & $\Delta \mathbf{G}$ & $\Delta \Delta \mathbf{G}$ \\
\hline \multirow[t]{2}{*}{$\Delta \mathbf{G}_{\text {ROT-TRANS }}$} & 0 & - & 0 & - \\
\hline & \multicolumn{2}{|c|}{ Minimal-HighSA } & \multicolumn{2}{|c|}{ Complete-LowSA } \\
\hline VOL-NP & -0.13 & -0.40 & 0.18 & 0.12 \\
\hline VOL-P & 0.94 & 2.30 & 0.25 & 0.91 \\
\hline $\mathbf{R}^{2}$ correlation & 0.43 & 0.61 & 0.43 & 0.49 \\
\hline
\end{tabular}

Weights are $w$ for $\Delta \mathrm{G}$ models, $\mathrm{w}^{\prime}$ for $\Delta \Delta \mathrm{G}$ models.

Measurements suggest that an entropy penalty corresponding to a free energy in the range of $0-10 \mathrm{~kJ}$ per mole of interacting species at $300 \mathrm{~K}$ is appropriate [25]. This is a relatively small range compared with measured $\Delta \mathrm{G}$ values (Table 1). Other computational work has set $\Delta \mathrm{G}_{\mathrm{ROT}}$ TRANS to zero [8]. We examine $\Delta \mathrm{G}_{\text {ROT-TRANS }}$ at either 0 or 10 $\mathrm{kJ} / \mathrm{mole}$ for each binding model, to test the sensitivity of the model to this term. Models calculated at these $\Delta \mathrm{G}_{\mathrm{ROT}}$ TRANS values, for a given set of conditions, are very similar (Table 2), so that the precise value is not a major consideration in this study. We are neglecting the entropic term associated with any changes in vibrational modes upon complexation [37].

Non-polar and polar solvent accessible surface areas are calculated with the program SACALC, developed in our laboratory. Areas are then differenced between a complex and the sum of its components, and multiplied by a factor of $0.1 \mathrm{~kJ} / \mathrm{mole} / \AA^{2}$. This factor is within the range generally assumed to represent the energetics of hydrophobic solvation in empirical modelling $[38,39]$. The precise value is not critical, since linear fitting will scale surface area terms via the pre-multipliers. The same factor of 0.1 has been used for polar surface area, and we include this feature in part to allow for some approximate recognition of hydrogen-bonding potential. Detailed assessment of pairwise hydrogen bonds, outside of the context of ionisable groups, is not included. Additionally, explicit van der Waals interactions are excluded. Both hydrogen bond and van der Waals interactions, with a strong distancedependence over fractions of an $\AA$, would be more appropriate if energy minimisation were carried out subsequent to sidechain repacking from a discrete rotamer library. We chose to develop binding models without energy minimisation, and this relatively simple approach gives a broad insight into differences between $\Delta \mathrm{G}$ and $\Delta \Delta \mathrm{G}$ models. Both the non-polar and polar area terms, as defined, are favourable for complexation when weights are positive.

Ionisable group interactions were derived from the $\mathrm{pH}$ dependence of electrostatic energy, with addition of a constant of integration at an extreme $\mathrm{pH}$ [40]. The $\mathrm{pH}$ dependent electrostatics were calculated with in-house programs using the FD/DH method that combines Finite Difference Poisson-Boltzmann (FDPB, [41]) and DebyeHûckel (DH) interaction schemes [42]. Monte Carlo sampling [43] was used to derive pKas [44] and the ionisable charge distribution, from which electrostatic energy is obtained [45]. Ionisable group energies are included with the $\Delta \mathrm{G}_{\text {IONIS-FDDH }}$ term, and may be either favourable or unfavourable for complexation.

We have previously introduced an empirical term to account for the entropy of water liberation upon ionisable group burial, using a comparison of calculated and measured pKas [46]. This analysis looked at hydration differences between ionised and neutral states. The significance of this empirical parameter in pKa calculations was generally small, which was rationalised in terms of little overall difference between water ordering in the ionised and neutral states. Cysteine was an exception, consistent with relatively little charge separation and reduced hydrogenbonding potential in the neutral state. The $\Delta \mathrm{G}_{\text {IONIS-DESOLV }}$ term in the current study models change in burial of ionisable groups upon complexation, rather than ionised/ neutral form differences. In order to model the entropy associated with hydration shell liberation (desolvation) for ionisable groups, we used the derived cysteine value from our previous work, since in this case the neutral form has relatively little charge separation. Then $\Delta \mathrm{G}_{\text {IONIS-DESOLV }}$ is this complete hydration shell value multiplied by the change in hydration shell volume (calculated from FDPB grids) upon complexation, with no other variation between ionisable group types. As with other terms, the weight derived by fitting to experimental data will indicate relative importance. With liberation of solvating water upon complexation, this term is expected to be favourable for binding.

Finally in the binding scheme is modelling of free energy changes due to alteration of sidechain rotameric entropy upon complexation, $\Delta \mathrm{G}_{\mathrm{SC} \text {-ROT }}$. We use a method based on mean-field packing of sidechains, with derived probabilities (p) for rotamers based on packing opportunities $[21,22]$. Sidechain rotameric entropy for each amino acid is the sum over $\mathrm{p}^{*} \ln (\mathrm{p})$ for rotamers. Values are summed over amino acids in a protein, differenced between free and complexed states, and multiplied by RT $=2.5 \mathrm{~kJ} / \mathrm{mole}$ to give a free energy contribution, prior to weighting by the pre-multiplier. Changes in sidechain rotameric entropy are expected to be unfavourable for complexation (restriction of sidechains).

We used two variations of the sidechain rotamer term. In the first, rotamer packing differences upon complexation 
are calculated as described, with interfacial sidechains free to explore different packings, even though energy calculation for other terms is based on a single conformer. This variation is termed "Unlocked". In the second, interfacial sidechains (in the complex) are modelled as fixed in the conformation used for other components of the energy calculation. Entropy changes will therefore be larger for the second case, which is described as "Locked". These differences are discussed in the Results section, although generally the impact of the sidechain rotameric entropy was low in the models, whichever scheme was used for interfacial flexibility.

\section{Binding differences model}

We simply difference the binding model between wildtype and mutant systems $\left(\Delta \Delta \mathrm{G}_{\mathrm{EXP}}=\Delta \mathrm{G}_{\mathrm{EXP}}[\mathrm{MUT}]-\Delta \mathrm{G}_{\mathrm{EXP}}\right.$ [WT]), with the rigid-body rotation and translation term cancelling. A prime has been added to the weights, demonstrating that we are allowing different fits for $\Delta G$ and $\Delta \Delta \mathrm{G}$ models.

$$
\begin{gathered}
\Delta \Delta \mathrm{G}_{\text {CALC }}=\mathrm{w}_{\text {ASA-NP }}^{\prime} \Delta \Delta \mathrm{G}_{\text {ASA-NP }}+\mathrm{w}_{\text {ASA-P }}^{\prime} \Delta \Delta \mathrm{G}_{\text {ASA-P }}+\mathrm{w}_{\text {IONIS- }}^{\prime} \\
\text { FDDH }^{\prime} \Delta \Delta \mathrm{G}_{\text {IONIS-FDDH }}+\mathrm{W}_{\text {IONIS }}^{\prime}=\text { DESOLV }^{\prime} \Delta \Delta \mathrm{G}_{\text {IONIS-DESOLV }}{ }^{+} \\
\mathrm{W}_{\text {SC-ROT }}^{\prime} \Delta \Delta \mathrm{G}_{\text {SC-ROT }}
\end{gathered}
$$

\section{Model fitting}

Multiple linear regression was used to determine the best fit $\Delta \mathrm{G}_{\mathrm{CALC}}$ and $\Delta \Delta \mathrm{G}_{\mathrm{CALC}}$ models to experiment. This regression was performed with the built-in least squares function of the GNU Regression, Econometrics and Timeseries Library package (GRETL, [47]). The Solver function in Microsoft Excel was also used to carry out linear regression. Weights are expected to be positive to make physical sense, other than for $\Delta \mathrm{G}_{\text {IONIS-FDDH, which could be of }}$ either sign with attractive or repulsive interactions to the fore.

\section{Sidechain repacking}

Starting with an experimental structure for a wild-type complex, there are questions of conformational change and repacking for the uncomplexed components and mutated proteins. Data selection should have eliminated systems with large-scale mainchain conformational changes, but sidechain rearrangement remains an issue.

The basis of our methodology is a mean-field program developed [21] from earlier work [22]. This uses pairwise packing of rotamers to derive probabilities for rotamers within a sidechain, according to an allowed van der Waals tolerance in the packing. Higher rotamer probability means coexistence with a larger number of neighbouring sidechain rotamers, and larger solvent accessibility (HighSA). Conversely, the lower (but non-zero) probability rotamers will tend to have lower solvent accessibility (LowSA). Both of these packing schemes/rotamer selec- tions are used in our studies of $\Delta \mathrm{G}$ and $\Delta \Delta \mathrm{G}$ models. Another question for sidechain repacking is whether to remain as close as possible to experimental (complex) structure (Minimal repacking), or whether to allow sidechain relaxations in response to complex separation and mutation (Complete repacking).

\section{Volume-based solvation}

A simple volume-based solvation function was used to replace the ASA-based analysis at some points in the work. Thus, $\mathrm{w}_{\text {VOL-NP }} \Delta \mathrm{G}_{\mathrm{VOL}-\mathrm{NP}}+\mathrm{w}_{\text {VOL-P }} \Delta \mathrm{G}_{\mathrm{VOL}-\mathrm{P}}$ was swapped into the $\Delta \mathrm{G}_{\mathrm{CALC}}$ equation in place of $\mathrm{w}_{\mathrm{ASA}-\mathrm{NP}} \Delta \mathrm{G}_{\mathrm{ASA}-\mathrm{NP}}+\mathrm{w}_{\mathrm{ASA}}$ ${ }_{\mathrm{p}} \Delta \mathrm{G}_{\mathrm{ASA}-\mathrm{p}}$, with the analogous weighted terms also for the $\Delta \Delta \mathrm{G}_{\mathrm{CALC}}$ binding model. These volume solvation terms are based on grid calculations of volumes around nonhydrogen atoms that are filled or unfilled by neighbouring atoms. Non-polar atoms and radii $(\AA)$ are C:2.0 and $\mathrm{S}: 1.9$. Polar atoms and radii are $\mathrm{O}: 1.5$ and $\mathrm{N}: 1.8$. Volumes for each atom are calculated with a $0.5 \AA$ spaced grid and a shell of thickness $2.8 \AA$ beyond the atomic radius. These calculations give numbers of grid points that are not covered by neighbouring atoms. In order to put the numbers onto a scale roughly equating to that for ASA-based terms, a multiplicative factor applied to the number of grid points in the solvation shell of a $\mathrm{C}$ atom was equated with the ASA energy for the same, unoccluded, atom. This multiplicative factor is 0.0042 .

\section{Availability and requirements}

Project name: Intcalc

Project home page: http://www.bioinf.manchester.ac.uk/ intcalc/

The software used in this study is also available for download from: http://personalpages.manchester.ac.uk/staff/ j.warwicker/resources.html

Operating system(s): Linux

Programming language: Perl, Fortran

\section{License: GNU GPL}

No additional restrictions for non-academic users.

\section{Abbreviations}

ASA: Accessible Surface Area; PDB: Protein Data Bank; PINT: Protein-protein Interactions Database; ASEdb: Alanine Scanning Energetics database; BPTI: Bovine Pancreatic Trypsin Inhibitor; FDPB: Finite Difference PoissonBoltzmann; DH: Debye-Hückel; FD/DH: Finite Difference/Debye-Hückel; GRETL: GNU Regression: Econometrics and Time-series Library. 


\section{Authors' contributions}

SB and JW conceived the study, interpreted the data and wrote the final manuscript together. SB and JW both contributed source code, whilst dataset creation and implementation of the computational analyses were due to SB.

\section{Acknowledgements}

The Algerian Ministry of Higher Education is thanked for the award of PhD funding to SB. We thank Dr Nick Gresham for technical support, and James Kitchen, Tracey Bray and Pedro Chan for discussions.

\section{References}

I. Auerbach D, Thaminy S, Hottiger MO, Stagljar I: The post-genomic era of interactive proteomics: facts and perspectives. Proteomics 2002, 2:61 I-623

2. Gingras AC, Aebersold R, Raught B: Advances in protein complex analysis using mass spectrometry. J Physiol 2005, 563: ||-2|.

3. Zhu X, Gerstein M, Snyder M: Getting connected: analysis and principles of biological networks. Genes Dev 2007, 2 I:1010-1024.

4. Berman HM, Westbrook J, Feng Z, Gilliland G, Bhat TN, Weissig H, Shindyalov IN, Bourne PE: The Protein Data Bank. Nucl Acids Res 2000, 28:235-242.

5. Chandonia J-M, Brenner SE: The impact of structural genomics: Expectations and outcomes. Science 2006, 3 I I :347-35 I.

6. Ritchie DW: Recent progress and future directions in proteinprotein docking. Curr Protein Pept Sci 2008, 9:I-I5.

7. Vakser IA, Kundrotas P: Predicting 3D structures of proteinprotein complexes. Curr Pharm Biotechnol 2008, 9:57-66.

8. Audie J, Scarlata S: A novel empirical free energy function that explains and predicts protein-protein binding affinities. Biophys Chem 2007, I29:198-2II.

9. Eisenstein M, Katchalski-Katzir E: On proteins, grids, correlations, and docking. C R Biol 2004, 327:409-420.

I0. Vajda S, Camacho Cl: Protein-protein docking: is the glass halffull or half-empty? Trends Biotechnol 2004, 22(3): I I0-I 16.

II. Gabb HA, Jackson RM, Sternberg MJ: Modelling protein docking using shape complementarity, electrostatics and biochemical information. J Mol Biol 1997, 272:106-120.

12. Althaus $E$, Kohlbacher O, Lenhof HP, Müller P: A combinatorial approach to protein docking with flexible side chains. J Comput Biol 2002, 9:597-6I2.

13. Schueler-Furman O, Wang C, Baker D: Progress in protein-protein dcking: atomic resolution predictions in the CAPRI experiment using RosettaDock with an improved treatment of side-chain flexibility. Proteins 2005, 60:187-194.

14. Wang C, Schueler-Furman O, Sndre I, London N, Fleishman SJ, Bradley $\mathrm{P}, \mathrm{Qian} \mathrm{B}$, Baker D: RosettaDock in CAPRI rounds 6-I 2. Proteins 2007, 69:758-763.

15. Aloy P, Russell RB: Ten thousand interactions for the molecular biologist. Nat Biotechnol 2004, 22:1317-|321.

16. Kiel C, Beltrao P, Serrano L: Analyzing protein interaction networks using structural information. Annu Rev Biochem 2008, 77:4I5-44I.

17. Horton N, Lewis M: Calculation of the free-energy of association for protein complexes. Protein Science 1992, I:I69-181.

18. Teng S, Michonova-Alexova E, Alexov E: Approaches and resources for prediction of the effects of non-synonymous single nucleotide polymorphism on protein function and interactions. Curr Pharm Biotechnol 2008, 9:123-133.

19. Guerois R, Nielsen JE, Serrano L: Predicting changes in the stability of proteins and protein complexes: a study of more than 1000 mutations. J Mol Biol 2002, 320:369-387.

20. Kortemme T, Baker D: A simple model for binding energy hot spots in protein-protein complexes. Proc Natl Acad Sci USA 2002, 99:|4|||6-|4|2|.

21. Cole C, Warwicker J: Side-chain conformational entropy at protein-protein interfaces. Protein Science 2002, I I :2860-2870.

22. Koehl P, Delarue M: Application of a self-consistent mean field theory to predict protein side-chains conformation and esti- mate their conformational entropy. I Mol Biol I994, 239:249-275

23. Xiang Z, Steinbach PJ, Jacobson MJ, Friesner RA, Honig B: Prediction of side-chain conformations on protein surfaces. Proteins 2007 , 66:8|4-823

24. Canutescu AA, Shelenkov AA, Dunbrack RL Jr: A graph-theory algorithm for rapid protein side-cahin prediction. Protein Science 2003, I 2:2001-2014.

25. Tamura A, Privalov PL: The entropy cost of protein association. J Mol Biol 1997, 273: I048-1060.

26. Vaughan CK, Buckle AM, Fersht AR: Structural response to mutation at a protein-protein interface. I Mol Biol 1999 , 286: | 487-I506.

27. Kang YK, Nemethy G, Scheraga HA: Free energies of hydration of solute molecules I. Improvement of the hydration shell model by exact computations of overlap volumes. J Chem Phys 1987, $91: 4105-4109$.

28. Colonna-Cesari F, Sander C: Excluded volume approximation to protein-solvent interaction. The solvent contact model. Biophys J 1990, 57: I 103-I I07.

29. Lazaridis $T$, Karplus $M$ : Effective energy function for proteins in solution. Proteins 1999, 35:| $33-152$.

30. Burgoyne NJ, Jackson RM: Predicting protein interaction sites: binding hot-spots in protein-protein and protein-ligand interfaces. Bioinformatics 2006, 22:1335-1342.

31. Wang T, Tomic S, Gabdoulline RR, Wade RC: How optimal are the binding energetics of barnase and barstar? Biophys J 2004, 87:1618-1630.

32. Thorn KS, Bogan AA: ASEdb: a database of alanine mutations and their effects on the free energy of binding in protein interactions. Bioinformatics 2001, I 7:284-285.

33. Kumar MD, Gromiha MM: PINT: Protein-protein Interactions Thermodynamic Database. Nucl Acids Res 2006, 34:DI95-DI 98.

34. Bava KA, Gromiha MM, Uedaira H, Kitajima K, Sarai A: ProTherm, version 4.0; thermodynamic database for proteins and mutants. Nucl Acids Res 2004, 32:DI20-DI2I.

35. Finkelstein AV, Janin J: The price of lost freedom: the entropy of bimolecular complex formation. Protein Eng 1989, 3:1-3.

36. Amzel LM: Loss of translational entropy in binding, folding, and catalysis. Proteins |997, 28: |44-|49.

37. Gohlke H, Case DA: Converging free energy estimates: MMPB(GB)SA studies on the protein-protein complex Ras-Raf. J Comput Chem 2004, 25:238-250.

38. Reynolds JA, Gilbert DB, Tanford C: Empirical correlation between hydrophobic free energy and aqueous cavity surface area. Proc Natl Acad Sci USA 1974, 71:2925-2927.

39. Lee $\mathrm{B}$ : Calculation of volume fluctuations for globular protein models. Proc Natl Acad Sci USA 1983, 80:622-626.

40. Chan P, Lovric J, Warwicker J: Subcellular $\mathbf{p H}$ and the predicted pH-dependent features of proteins. Proteomics 2006, 6:3494-350I.

4I. Warwicker J: Continuum dielectric modelling of the proteinsolvent system, and calculation of the long-range electrostatic field of the enzyme phosphoglycerate mutase. J Theor Biol 1986, I 2 I: 199-2 I0.

42. Warwicker J: Improved pKa calculations through flexibility based sampling of a water-dominated interaction scheme. Protein Science 2004, I 3:2793-2805.

43. Beroza P, Fredkin DR, Okamura MY, Feher G: Protonation of interacting residues in a protein by a Monte Carlo method: application to lysozyme abd the photosynthetic reaction center of Rhodobacter sphaeroides. Proc Natl Acad Sci USA 1991, 88:5804-5808.

44. Bashford D, Karplus : pKa's of ionisable groups in proteins: atomic detail from a continuum electrostatic model. Biochemistry 1990, 29:10219-10225.

45. Antosiewicz J, McCammon JA, Gilson MK: Prediction of the $\mathbf{p H}$ dependent properties of proteins. J Mol Biol 1994, 238:4I5-436.

46. Warwicker J: Improving pKa calculations with consideration of hydration entropy. Protein Eng 1997, 10:809-8|4.

47. Gnu Regression, Econometrics and Time-series Library [http://gretl.sourceforge.net/]

48. Guex N, Peitsch MC: Swiss-Model and the Swiss-PdbViewer: an environment for comparative protein modelling. Electrophoresis 1997, I 8:27|4-2723. 
49. Castro MJM, Anderson S: Alanine point-mutations in the reactive region of bovine pancreatic trypsin inhibitor: Effects on the kinetics and thermodynamics of binding to beta-trypsin and alpha-chymotrypsin. Biochemistry 1996, 35: | | 435- I I 446.

50. Frisch C, Schreiber G, Johnson CM, Fersht AR: Thermodynamics of the interaction of barnase and barstar: changes in free energy versus changes in enthalpy on mutation. I Mol Biol 1997, 267:696-706.

5I. Horn JR, Ramaswamy S, Murphy KP: Structure and Energetics of Protein-Protein Interactions: The Role of Conformational Heterogeneity in OMTKY3 Binding to Serine Proteases. J Mol Biol 2003, 33 I:497-508.

52. Herrmann C, Horn G, Spaargaren M, Wittinghofer A: Differential Interaction of the Ras Family GTP-binding Proteins H-Ras, Rap IA, and R-Ras with the Putative Effector Molecules Raf Kinase and Ral-Guanine Nucleotide Exchange Factor. J Biol Chem 1996, 27I:6794-6800.

53. Wohlgemuth S, Kiel C, Krämer A, Serrano L, Wittinghofer F, Herrmann C: Recognizing and Defining True Ras Binding Domains I: Biochemical Analysis. J Mol Biol 2005, 348:74I-758.

54. Dall'Acqua W, Goldman ER, Eisenstein E, Mariuzza RA: A mutational analysis of the binding of two different proteins to the same antibody. Biochemistry 1996, 35:9667-9676.

55. Verhoeyen M, Milstein C, Winter G: Reshaping human antibodies: Grafting an antilysozyme activity. Science 1988 , 239: $1534-1536$

56. Rajpal A, Taylor MG, Kirsch JF: Quantitative evaluation of the chicken lysozyme epitope in the HyHEL- 10 Fab complex: Free energies and kinetics. Protein Science 1998, 7:1868-1874.

57. Kiel C, Serrano L, Herrmann C: A Detailed Thermodynamic Analysis of Ras/Effector Complex Interfaces. I Mol Biol 2004, 340: 1039-1058.

58. Ascenzi P, Amiconi G, Menegatti E, Guarneri M, Bolognesi M, Schnebli HP: Binding of the recombinant proteinase inhibitor eglin $c$ from leech Hirudo medicinalis to human leukocyte elastase, bovine alpha-chymotrypsin and subtilisin Carlsberg: Thermodynamic study. J Enzyme Inhib 1988, 2(3): 167-I72.

59. Wallis R, Leung KY, Osborne MJ, James R, Moore GR, Kleanthous C: Specificity in Protein-Protein Recognition: Conserved Im9 Residues Are the Major Determinants of Stability in the Colicin E9 DNase-Im9 Complex. Biochemistry 1998, 37:476-485.

60. Lavoie TB, Drohan WN, Smith-Gill S): Experimental analysis by site-directed mutagenesis of somatic mutation effects on affinity and fine specificity in antibodies specific for lysozyme. J Immunol 1992, 148:503-513.

61. Read RJ, Fujinaga M, Sielecki AR, James MNG: Structure of the complex of Streptomyces griseus protease $B$ and the third domain of the turkey ovomucoid inhibitor at I.8 A resolution. Biochemistry 1983, 22:4420-4433.

62. Shapiro R, Ruiz-Gutierrez M, Chen CZ: Analysis of the interactions of human ribonuclease inhibitor with angiogenin and ribonuclease $A$ by mutagenesis: Importance of inhibitor residues inside versus outside the $\mathbf{C}$-terminal 'hot spot'. J Mol Biol 2000, 302:497-5I9.

63. Kortt AA, Nice E, Gruen LC: Analysis of the Binding of the Fab Fragment of Monoclonal Antibody NClO to Influenza Virus N9 Neuraminidase from Tern and Whale Using the BIAcore Biosensor: Effect of Immobilization Level and Flow Rate on Kinetic Analysis. Anal Biochem 1999, 273:I33-I4I.

64. Uehara $Y$, Tonomura B, Hiromi K: Direct fluorometric determination of a dissociation constant as low as 10-10 $\mathrm{M}$ for the subtilisin BPN'-protein proteinase inhibitor (Streptomyces subtilisin inhibitor) complex by a single photon counting technique. J Biochem 1978, 84: I 195-1202.

65. Pineda AO, Cantwell AM, Bush LA, Rose T, Cera ED: The thrombin epitope recognizing thrombomodulin is a highly cooperative hot spot in exosite I. J Biol Chem 2002, 277:32015-32019.

66. Chen Z, Bode W: Refined 2.5 ^ X-ray crystal structure of the complex formed by porcine kallikrein $A$ and the bovine pancreatic trypsin inhibitor. Crystallization, Patterson search, structure determination, refinement, structure and comparison with its components and with the bovine trypsin-pancreatic trypsin inhibitor complex. J Mol Biol |983, I64:283-3| I.

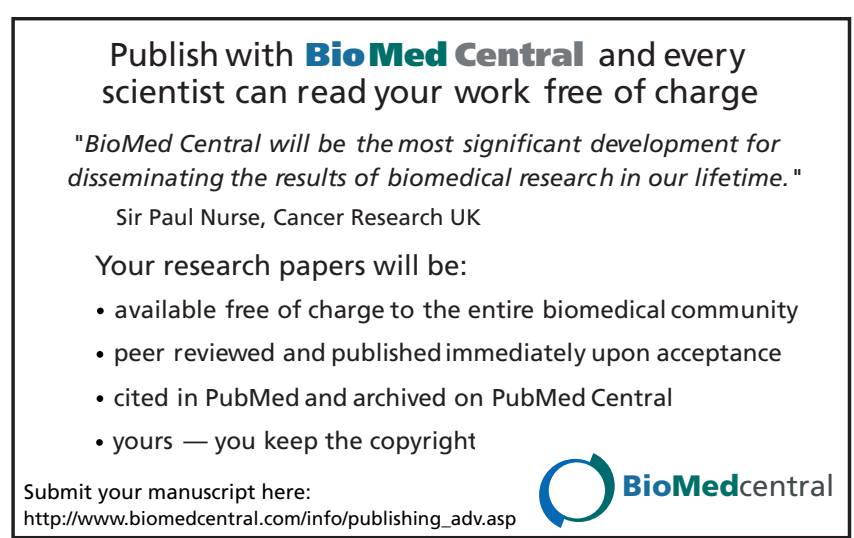

